\title{
Obsessions or psychosis?
}

Donald W. Simpson II, MD, Izza Bazigh, MBBS, and Rekha Bandla Kasi, DO

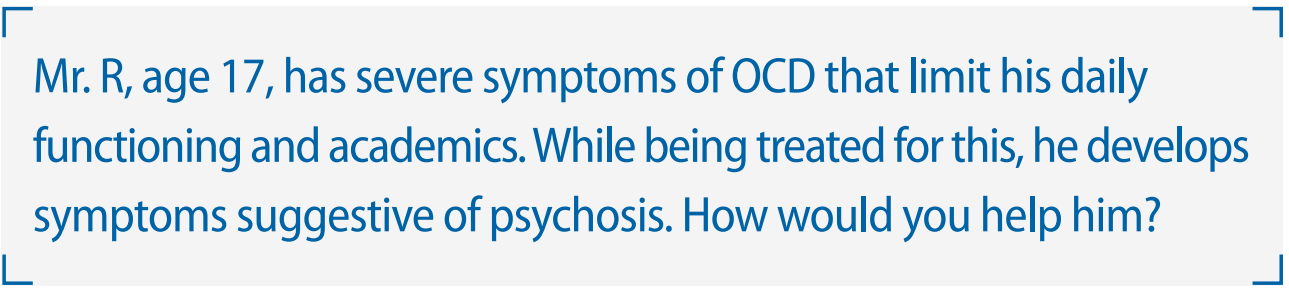

\section{CASE Perseverating on}

\section{nonexistent sexual assaults}

Mr. R, age 17, who has been diagnosed with obsessive-compulsive disorder (OCD), presents to the emergency department (ED) because he thinks that he is being sexually assaulted and is concerned that he is sexually assaulting other people. His family reports that Mr. R has perseverated over these thoughts for months, although there is no evidence to suggest these events have occurred. In order to ameliorate his distress, he performs rituals of looking upwards and repeatedly saying, "It didn't happen."

Mr. $R$ is admitted to the inpatient psychiatry unit for further evaluation.

\section{HISTORY Decompensation while attending a PHP}

Mr. R had been diagnosed with bipolar disorder and attention-deficit/hyperactivity disorder when he was 13 . During that time, he was treated with divalproex sodium and dextroamphetamine. At age 15, Mr. R's diagnosis was changed to OCD. Seven months before coming to the $E D$, his symptoms had been getting worse. On one occasion, Mr. R was talking in a nonsensical fashion at school, and the police were called. Mr. $\mathrm{R}$ became physically aggressive with the police and was subsequently hospitalized, after which he attended a partial hospitalization program (PHP). At the PHP,
Mr. R received exposure and response prevention therapy for $O C D$, but did not improve, and his symptoms deteriorated until he was unable to brush his teeth or shower regularly. While attending the PHP, Mr. R also developed disorganized speech. The PHP clinicians became concerned that Mr. R's symptoms may have been prodromal symptoms of schizophrenia because he did not respond to the OCD treatment and his symptoms had worsened over the 3 months he attended the PHP.

\section{EVALUATION Normal laboratory results}

Upon admission to the inpatient psychiatric unit, Mr. R is restarted on his home medications, which include fluvoxamine, $150 \mathrm{mg}$ in the morning and $200 \mathrm{mg}$ at bedtime; methylfolate, $2,000 \mathrm{mcg} / \mathrm{d}$; risperidone, $3 \mathrm{mg}$ nightly; and hydroxyzine, $25 \mathrm{mg}$ as needed for anxiety.

His laboratory workup, including a complete blood count, comprehensive metabolic

Dr. Simpson is a Child and Adolescent Psychiatry Fellow, Rush University Medical Center, Chicago, Illinois. Dr. Bazigh is a Child and Adolescent Psychiatry Research Observer, Rush University Medical Center, Chicago, Illinois. Dr. Kasi is an Assistant Professor, Department of Psychiatry and Behavioral Sciences, Rush University Medical Center, Chicago, Illinois.

Disclosures

The authors report no financial relationships with any companies whose products are mentioned in this article, or with manufacturers of competing products.

doi: $10.12788 / \mathrm{cp} .0042$

\section{How would you handle this case?}

Answer the challenge questions at MDedge.com/ psychiatry and see how your colleagues responded 


\section{Clinical Point}

\section{There appears to be both an epidemiologic and biologic overlap between $O C D$ and schizophrenia}

panel, urine drug screen, and blood ethanol, are all within normal limits. Previous laboratory results, including a thyroid function panel, vitamin D level, and various autoimmune panels, were also within normal limits.

His family reports that Mr. R's symptoms seem to worsen when he is under increased stress from school and prepping for standardized college admission examinations. The family also says that while he is playing tennis, Mr. $\mathrm{R}$ will posture himself in a crouched down position and at times will remain in this position for 30 minutes.

Mr. R says he eventually wants to go to college and have a professional career.

\section{What should be considered in the differential diagnosis for Mr. R? \\ a) OCD with absent insight/delusional beliefs \\ b) OCD with comorbid schizophrenia \\ c) bipolar disorder \\ d) psychotic disorder due to another medical condition \\ e) all of the above}

\section{The authors' observations}

When considering Mr. R's diagnosis, our treatment team considered the possibility of OCD with absent insight/delusional beliefs, OCD with comorbid schizophrenia, bipolar disorder, and psychotic disorder due to another medical condition.

\section{Overlap between OCD and schizophrenia}

There appears to be both an epidemiologic and biologic overlap between OCD and schizophrenia. The Table ${ }^{1}$ (page 49) summarizes the DSM- 5 criteria for OCD and for schizophrenia. Schirmbeck et al ${ }^{2}$ reported that the estimated prevalence of OCD in patients with schizophrenia is $12 \%$, which is higher than in the general population. Obsessivecompulsive symptoms (OCS) in patients with schizophrenia have been reported to be even more prevalent $(30.7 \%)^{2}$ In a prospective cohort study, de Haan et al ${ }^{3}$ assessed 172 patients with first-episode schizophrenia, schizophreniform disorder, or schizoaffective disorder for the development of OCS over a 5-year follow-up period. Symptoms were tracked over time and included OCS on first assessment, persistent OCS, subsequent emergence of OCS, and intermittent OCS. A striking $51.1 \%$ of the patient sample screened positive. ${ }^{3}$ Obsessions and delusions are similar because they are both irrational thoughts, the former with insight and the latter without insight. The fact that OCS were present in up to $14 \%$ of drug-naïve patients with schizophrenia in this study suggests that this was not merely an adverse effect of antipsychotic medication.

Much of the literature about OCD examines its functional impairment in adults, with findings extrapolated to pediatric patients. Children differ from adults in a variety of meaningful ways. Baytunca et $\mathrm{al}^{4}$ examined adolescents with early-onset schizophrenia, with and without comorbid OCD. Patients with comorbid OCD required higher doses of antipsychotic medication to treat acute psychotic symptoms and maintain a reduction in symptoms. The study controlled for the severity of schizophrenia, and its findings suggest that schizophrenia with comorbid OCD is more treatment-resistant than schizophrenia alone. ${ }^{4}$

Some researchers have theorized that in adolescents, OCD and psychosis are integrally related such that one disorder could represent a prodrome or a cause of the other disorder. Niendam et al ${ }^{5}$ studied OCS in the psychosis prodrome. They found that OCS can present as a part of the prodromal picture in youth at high risk for psychosis. However, because none of the patients experiencing OCS converted to full-blown psychosis, these results suggest that OCS may not represent a prodrome to psychosis per se. Instead, these individuals may represent a subset of false positives over the follow-up period. ${ }^{5}$ Another possible explanation for the increased emergence of pre-psychotic symptoms in adolescents 


\section{DSM-5 diagnostic criteria: OCD vs schizophrenia}

\section{OCD}

The presence of obsessions, compulsions, or both

Obsessions are defined by (1) and (2):

(1) recurrent and persistent thoughts, urges, or impulses that are experienced, at some time during the disturbance, as intrusive and unwanted, and that in most individuals cause marked anxiety or distress

(2) the individual attempts to ignore or suppress such thoughts, urges, or images, or to neutralize them with some other thought or action

Compulsions are defined by (1) and (2):

(1) repetitive behaviors or mental acts that the individual feels driven to perform in response to an obsession or according to rules that must be applied rigidly

(2) the behaviors or mental acts are aimed at preventing or reducing anxiety or distress, or preventing some dreaded event or situation; however, these behaviors or mental acts are not connected in a realistic way with what they are designed to neutralize or prevent, or are clearly excessive

OCD: obsessive-compulsive disorder

Source: Reference 1

\section{Schizophrenia}

The presence of $\geq 2$ of the following for at least 1 month, and at least 1 of them must be the first 3 :

(1) delusions

(2) hallucinations

(3) disorganized speech

(4) grossly disorganized or catatonic behavior

(5) negative symptoms, such as diminished emotional expression

Some signs of the disorder must last for a continuous period of at least 6 months with OCD could be a difference in their threshold of perception. OCS compels adolescents with OCD to self-analyze more critically and frequently. As a result, these patients may more often report depressive symptoms, distress, and exacerbations of pre-psychotic symptoms. These findings highlight that comorbid OCD can amplify the psychosocial distress among higher-risk youth. It is therefore essential for physicians to perform a thorough interview in this population because subtle psychotic symptoms may be present.

\section{What would you consider as a first-line} treatment for an adolescent with symptoms of OCD and schizophrenia?
a) a tricyclic antidepressant and a first- generation antipsychotic
b) a selective serotonin reuptake inhibitor (SSRI) and a second-generation antipsy- chotic (SGA)
c) an SGA alone
d) an SSRI alone

\section{TREATMENT Improvement after switching to haloperidol}

The treatment team decides to change Mr. R's medications by cross-titrating risperidone to lurasidone and increasing hydroxyzine from 25 to $50 \mathrm{mg}$ every 6 hours as needed for anxiety. Over the next several days, Mr. R reports some improvement in symptoms. However, according to staff on the unit, he continues to display disorganized behavior, respond to internal stimuli, and posture in his room. It is unclear if these symptoms are due to a psychotic illness or part of his OCD rituals. Due to worsening of symptoms, the team decides to taper lurasidone and switch to haloperidol. Mr. R starts haloperidol, $1 \mathrm{mg}$ twice a day, and this is titrated to $7.5 \mathrm{mg}$ three times a day. Soon after, his thoughts become more organized, he has fewer delusional thoughts, his concentration is improved, and he no longer appears to respond to internal stimuli.

The treatment team obtains a consultation on whether electroconvulsive therapy would be appropriate, but this treatment is

\section{Clinical Point}

Some researchers have theorized that in adolescents, schizophrenia and OCD are integrally related 


\section{Clinical Point}

Mr. R's case highlights
how a psychiatric
diagnosis can produce
anxiety as a result
of the psychosocial
stressors associated
with that diagnosis

not recommended. Instead, the team considers switching Mr. $R$ to clozapine if the current treatment fails. Because Mr. R's psychotic symptoms continue to improve while he is receiving haloperidol, clozapine is not added. To address Mr. R's persistent, severe OCD symptoms, fluvoxamine is cross-tapered to sertraline, started at $50 \mathrm{mg} / \mathrm{d}$ and titrated to $100 \mathrm{mg} / \mathrm{d}$. Mr. R shows significant improvement in the days that follow.

Throughout admission, Mr. $R$ focuses on his lack of improvement and how this episode is negatively impacting his grades and his dream of going to college and having a professional career.

\section{OUTCOME Relief at last}

Mr. R improves with the addition of sertraline and tolerates rapid titration well. He continues haloperidol without adverse effects, and is discharged home with close follow-up in a PHP and outpatient psychiatry.

However, after discharge, Mr. R's symptoms get worse, and he is admitted to a different inpatient facility. At this facility, he continues sertraline, but haloperidol is crosstitrated to olanzapine.

Currently, Mr. R has greatly improved and is able to function in school. He takes sertraline, $100 \mathrm{mg}$ twice a day, and olanzapine, 7.5 $\mathrm{mg}$ twice a day. Mr. $\mathrm{R}$ reports his rituals have reduced in frequency to less than 15 minutes each day. His thought processes are organized, and he is confident he will be able to achieve his goals.

\section{The authors' observations}

Given Mr. R's rapid improvement once an effective pharmacologic regimen was established, we concluded that he had a severe case of OCD with absent insight/delusional beliefs, and that he did not have schizophrenia. Mr. R's case highlights how a psychiatric diagnosis can produce anxiety as a result of the psychosocial stressors and limitations associated with that diagnosis.

\footnotetext{
References

1. Diagnostic and statistical manual of mental disorders, 5th ed. Washington, DC: American Psychiatric Association; 2013.

2. Schirmbeck F, Swets M, de Haan L. Obsessive-compulsive symptoms in schizophrenia. In: De Haan L, Schirmbeck F, Zink M. Epidemiology: prevalence and clinical characteristics of obsessive-compulsive disorder and obsessive-compulsive symptoms in patients with psychotic disorders. New York, NY: Springer International Publishing; 2015:47-61.

3. de Haan L, Sterk B, Wouters L, et al. The 5-year course of obsessive-compulsive symptoms and obsessive-compulsive disorder in first-episode schizophrenia and related disorders. Schizophr Bull. 2011;39(1):151-160.

4. Baytunca B, Kalyoncu T, Ozel I, et al. Early onset schizophrenia associated with obsessive-compulsive disorder: clinical features and correlates. Clin Neuropharmacol. 2017;40(6):243-245.

5. Niendam TA, Berzak J, Cannon TD, et al. Obsessive compulsive symptoms in the psychosis prodrome: correlates of clinical and functional outcome. Schizophr Res. 2009;108(1-3):170-175.
}

\section{Bottom Line}

There is both an epidemiologic and biologic overlap between obsessive-compulsive disorder and schizophrenia. In adolescents, either disorder could represent a prodrome or a cause of the other. It is essential to perform a thorough assessment of individuals with obsessive-compulsive disorder because these patients may exhibit subtle psychotic symptoms. 